Annales Geophysicae (2002) 20: 69-80 C European Geophysical Society 2002

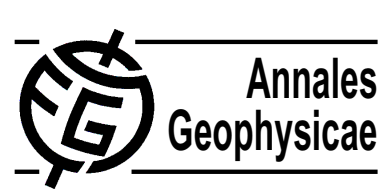

\title{
Low frequency modulation of transionospheric radio wave amplitude at low-latitudes: possible role of field line oscillations
}

\author{
A. K. Sinha, B. M. Pathan, R. Rajaram, and D. R. K. Rao \\ Indian Institute of Geomagnetism, Dr. Nanabhai Moos Marg, Colaba, Bombay 400 005, India
}

Received: 22 May 2001 - Revised: 9 August 2001 - Accepted: 17 September 2001

\begin{abstract}
Ionospheric scintillations of radio waves at lowlatitudes are associated with electron density irregularities. These irregularities are field-aligned and can provide excitation energy all along the field line to non-local field-aligned oscillations, such as the local field line oscillations. Eigenperiods of toroidal field line oscillations at low-latitudes, computed by using the dipole magnetic field and ion distributions obtained from the International Reference Ionosphere (IRI) for typical nighttime conditions, fall in the range of 20-25 s. When subjected to spectral analysis, signal strength of the radio waves recorded on the $250 \mathrm{MHz}$ beacon at Pondicherry $\left(4.5^{\circ} \mathrm{N}\right.$ dip), Mumbai $\left(13.4^{\circ} \mathrm{N}\right.$ dip) and Ujjain $\left(18.6^{\circ} \mathrm{N}\right.$ dip) exhibit periodicities in the same range. For the single event for which simultaneous ground magnetic data were available, the geomagnetic field also oscillated at the same periodicity. The systematic presence of a significant peak in the 20-25 s range during periods of strong radio wave scintillations, and its absence otherwise suggests the possibility that field line oscillations are endogenously excited by the irregularities, and the oscillations associated with the excited field line generate the modulation characteristics of the radio waves received on the ground. The frequency of modulation is found to be much lower than the characteristic frequencies that define the main body of scintillations, and they probably correspond to scales that are much larger than the typical Fresnel scale. It is possible that the refractive mechanism associated with larger scale long-lived irregularities could be responsible for the observed phenomenon. Results of a preliminary numerical experiment that uses a sinusoidal phase irregularity in the ionosphere as a refracting media are presented. The results show that phase variations which are large enough to produce a focal plane close to the ground can reproduce features that are not inconsistent with our observations.
\end{abstract}

Key words. Magnetospheric physics (magnetosphere ionosphere interactions) Ionosphere (ionosphere - magne-

Correspondence to: A. K. Sinha (ashwini@iig.iigm.res.in) toshere interactions; ionospheric irregularities)

\section{Introduction}

Recently (Fedorov, 1999), there has been a renewal of interest in the suggestion of Saito (1983) that the near equatorial region may not only be passively amplifying the current system imposed from elsewhere, but could also be an active source of the generation of ULF geomagnetic disturbances. This idea of Saito (1983) stemmed from the fact that there was an additional late evening maximum in the diurnal distribution of $\mathrm{Pc} 3$ pulsations that were observed only at stations close to the dip equator. Though Pc3 geomagnetic pulsations are supposed to be associated with hydromagnetic oscillations excited on geomagnetic field line, their characteristics at low- and equatorial-latitudes are not yet understood either observationally or theoretically (Yumoto, 1986). It should be noted that it is around these local times that the lowand equatorial-latitude ionosphere is manifested with what is known as the spread-F, which is associated with waves and turbulence covering a wide range of spatial and temporal scales and generated by a variety of instability processes that persist almost right through the night. The irregularities are field-aligned and can provide free energy all along the field line to stimulate the field line oscillation.

The concept of an independent field line oscillation was introduced by Dungey (1954) who proposed for the first time that the long-period geomagnetic pulsations might be the result of standing Alfvén waves being excited on geomagnetic field lines. The concept has thereafter taken deep roots and there are many ground-based and satellite observations that have established the relevance of field line oscillations (Cummings et al., 1969; Anderson et al., 1990). A number of theoretical estimates of field line eigen-periods are available (Cummings et al., 1969; Sinha and Rajaram, 1997), but most of these are valid for middle and high-latitude regions. The estimates of Poulter et al. (1988) are more relevant to lowand equatorial-latitudes. These were based on dipole mag- 
Table 1. Coordinates of stations

\begin{tabular}{lcccc}
\hline Station & Geog. Lat. & Geog. Long. & Geomag. Lat. & Geomag. Long. \\
\hline Tirunalvely & $8.7^{\circ} \mathrm{N}$ & $77.8^{\circ} \mathrm{E}$ & $0.32^{\circ} \mathrm{S}$ & $149.75^{\circ}$ \\
Pondicherry & $11.9^{\circ} \mathrm{N}$ & $79.9^{\circ} \mathrm{E}$ & $2.7^{\circ} \mathrm{N}$ & $152.1^{\circ}$ \\
Mumbai & $18.55^{\circ} \mathrm{N}$ & $72.54^{\circ} \mathrm{E}$ & $9.99^{\circ} \mathrm{N}$ & $145.64^{\circ}$ \\
Ujjain & $23.18^{\circ} \mathrm{N}$ & $75.78^{\circ} \mathrm{E}$ & $14.28^{\circ} \mathrm{N}$ & $149.21^{\circ}$ \\
\hline
\end{tabular}

netic fields and a physically realistic (but not a standard) ion density model. In Sect. 2, we cross check their results using a standard IRI model for the ion density.

In the satellite data (Anderson et al., 1990), a dynamic spectrum of geomagnetic field oscillations shows a clear increase in the frequency in the in-bound sector of the orbit and a decrease in the frequency in the out-bound sector, consistent with the expectations from the model of field line oscillations. Signatures of such oscillations have been detected by the AMPTE CCE satellite, right from 8 Earth radii to almost 2 Earth radii (Takahashi and Anderson, 1992). Field line oscillations in the ground geomagnetic data are determined by the frequency spectra at the foot of the field line. The signal corresponding to the characteristic eigen-period of the field line will maximize on the ground at the latitude corresponding to the foot of the field line (Menk et al., 1994). This period will thus change with latitude. The scenario is quite different, as we shall see later, when one is looking for signatures of field line oscillations in the radio wave signals recorded on the ground.

Radio wave scintillations recorded on the ground is one of the important consequences of the presence of irregularities in the F-region of the ionosphere at low-latitudes in the postdusk hours. Radio waves scattered by small-scale irregularities with scales typically less than the Fresnel scale (Bhattacharyya, 1999) form a diffraction pattern on the ground and as the irregularities drift in space, the received signals at a fixed station 'scintillate' with a time scale of a fraction of seconds with amplitudes (depth of scintillations) of the order of the received signal itself. The diffraction pattern on the ground is often treated under the phase screen approximation. The role of the ionosphere is visualized as that of a thin phase screen which introduces a shift of phase in the radio wave passing through it. The phase shift introduced is a function of a spatial coordinate on the screen through which the radio wave passes through. The phase shift introduced can thus be Fourier analyzed and the power Fourier components can be identified with the strength of the irregularities and the corresponding wavelengths can be identified with the scale size of the irregularities. Typical phase amplitudes associated with scale sizes less than the Fresnel scale are believed to be of the order of 1 radian (Yeh and Liu, 1982). A movement of the phase screen associated with the field line oscillation can provide a phase modulation, but it would appear that frequency modulation of such a small phase shift is unlikely to produce significant power at the period of the oscillation.
However, it has also been suggested that larger scales corresponding to the typical scale size in the ionosphere can also contribute as they may be associated with amplitudes of phase variation as high as hundreds to thousands of radians (Booker and Majidiahi, 1981). In this case, the irregularity acts as a refracting media and the interference of the refracted waves on the ground produce the spatial pattern whose movement causes scintillations in a fixed location radio wave record. For the right choice of phase shift amplitude and scale size, the refraction of radio waves can come to focus close to the ground plane (Titheridge, 1971) and even small movements of the irregularity pattern in the ionosphere can cause measurable changes in the received radio wave signal at a fixed station. Such movements can be provided by transverse field line oscillations.

Indian Institute of Geomagnetism operates receiving stations for recording radio wave signals from FLEETSAT (at $73^{\circ} \mathrm{E}$ ) at $250 \mathrm{MHz}$. The coordinates of the receiving stations are given in Table 1. The data base provides an excellent opportunity to examine whether there is any evidence of modulation through transverse oscillations in the received radio wave signals. The presence of ULF waves signatures in the radio waves has been detected earlier (Sutcliffe and Poole, 1989, 1990 and references therein) in the Doppler shift of received signals. However, the operating frequencies for such studies are typically in the region of $4-10 \mathrm{MHz}$ and at these frequencies, the refractive indices are sensitive to changes in the magnetic field and electron densities (Sutcliffe and Poole, 1990). A careful look at the expression of the refractive index at radio frequencies (Budden, 1961) shows that for $250 \mathrm{MHz}$, the refractive index is close to unity and the background density and magnetic field contribute less than a fraction of a percent. Therefore it is thus primarily the movement of irregularities that can contribute to any observed modulation and that is what one has to essentially look for. Support from simultaneous magnetic field data sampled at around one second is very useful but unfortunately not available. Data at a 5 second sampling has become available at Pondicherry only very recently and was only of limited use.

In Sect. 2, we compute the fundamental toroidal frequency for the Indian longitudinal sector by taking the relevant mass density and ionization from the IRI model. In Sect. 3, we look for the signatures of such pulsations in the ionospheric waves at low-latitude stations and the magnetic field data for the event for which they were available. Finally, in Sect. 4, we report the results of a simple numerical experiment that uses a sinusoidal phase irregularity in the ionosphere as a refracting media. The results show that phase variations which are large enough to produce a focal plane close to the ground can reproduce features that are consistent with our observations. The main conclusions are stated in Sect. 5.

\section{Theoretical estimates of toroidal eigen periods}

Theoretical estimates of field line eigen-periods are available (Cummings et al., 1969; Sinha and Rajaram, 1997). How- 
ever, most of such estimates are based on a suitably chosen density profile as a power law $r^{-m}$ for the hydrogen plasma. Such estimates are not valid for low-latitude regions, i.e. for lower L-values where the field lines are almost glued to the ionosphere and the most dominant species are $O^{+}$rather than $\mathrm{H}^{+}$. Poulter et al. (1988) used a dipole magnetic field and a specific but non-standard ion density model. These should be considered appropriate, but we reexamined the problem using a standard IRI model and computed the plasma loading along the geomagnetic field line. A model calculation for the typical fundamental toroidal mode eigen frequencies has been made numerically. The model is based on the dipolar field line geometry of the Earth's magnetic field and the field lines are held rigid at their ends in the infinitely conducting ionosphere represented by an F1 peak at a $200 \mathrm{~km}$ height from the Earth's surface. As noted earlier, the density of various species are taken from the International Reference Ionosphere model (site:http//www.nssdc.gsfc.nasa.gov/space/model). The reason for taking the infinitely conducting ionosphere at $200 \mathrm{~km}$ from the Earth's surface is that we could still neglect the collisional effect. The ratio of collision frequency of ion $\left(v_{i}\right)$ to the ion cyclotron frequency $\left(\Omega_{i}\right)$ at this height is $10^{-2}$ (Ratcliffe, 1972). Therefore, we could still work with collisionless approximation, thereby avoiding analytical complexities. In order to find the latitudinal profile of the fundamental toroidal eigen mode, the second order wave equation (Singer et al., 1981) has been considered. The equation describes the low frequency transverse waves in an infinitely conducting stationary magnetized plasma with zero pressure. The equation could be written as

$\mu_{o} \rho \frac{\partial^{2}}{\partial t^{2}}\left(\xi_{\alpha} / h_{\alpha}\right)=\frac{1}{h_{\alpha}^{2}} \boldsymbol{B}_{\boldsymbol{o}} \cdot \nabla\left[h_{\alpha}^{2} \boldsymbol{B}_{\boldsymbol{o}} \cdot \nabla\left(\xi_{\alpha} / h_{\alpha}\right)\right]$,

where $\mu_{o}$ is the magnetic permeability in the vacuum, $\rho$ is the plasma mass density, $\xi_{\alpha}$ is the plasma displacement perpendicular to the field line, $\boldsymbol{B}_{\boldsymbol{o}}$ is the ambient magnetic field, the parameter $\alpha$ signifies the mode of oscillation and determines the direction $\boldsymbol{\alpha}$ of the field line displacement, and $h_{\alpha}$ is the scale factor for the normal separation between the field lines in the direction $\boldsymbol{\alpha}$ and is determined by the ambient magnetic field structure.

We solve Eq. (1) under the following assumptions:

(a) The ambient magnetic field is dipolar in nature;

(b) The time dependence of all the perturbed quantities is of the form $\exp [\iota \omega t]$;

(c) The field lines are rigidly fixed at their ends in the ionosphere.

If $s$ is the distance measured along the field line, Eq. (1) under the above assumptions takes the following dimensionless form

$$
-\Omega^{2} \rho^{\prime} X=\frac{B}{h_{\alpha}^{2}} \frac{d}{d S}\left[h_{\alpha}^{2} B \frac{d X}{d S}\right]
$$

with

$S=\frac{s}{R_{E}}, \Omega=\frac{\omega R_{E}}{V_{\text {Aeq }}}, X=\frac{\xi_{\alpha}}{R_{E} h_{\alpha}}, B=\frac{B_{o}}{B_{e q}}, \rho^{\prime}=\frac{\rho}{\rho_{e q}},(3)$

where $R_{E}$ is the Earth's radius, $V_{\text {Aeq }}$ is the Alfvén velocity at the equator, $B_{e q}$ is the ambient field at the equator, and $\rho_{e q}$ is the mass density of the plasma at the equator.

It should be noted that under the dipole approximation $h_{\alpha}$, $B$ and $B_{e q}$ are given as

$h_{\alpha}=L \sin ^{3} \theta$

for the toroidal mode, $\theta$ being the co-latitude.

$B=\frac{\sqrt{1+3 \cos ^{2} \theta}}{\sin ^{6} \theta}$

and

$B_{e q}=\frac{0.311}{L^{3}}$ Gauss,

where $L=1 / \sin ^{2} \theta_{c}$ (suffix $c$ indicates the value at the foot of the field line in the ionosphere) is the geocentric distance in the unit of the Earth's radius of the point where the field line crosses the equatorial plane.

Equation (2) can be solved numerically by decoupling it into two first order equations

$\frac{d X}{d S}=\frac{1}{h_{\alpha}^{2} B} Y$

$\frac{d Y}{d S}=-\frac{\Omega^{2} h_{\alpha}^{2}}{B} \rho^{\prime} X$.

The solution of the coupled Eqs. (4) and (5) was obtained using the second order Runge-Kutta method and the frequency $\omega$ was estimated by a shooting method using the boundary condition that the eigen function $X$ should vanish at the conjugate points.

There are certain points to be noted here. The density and relative ionization profile of various species chosen in this calculation have been taken from the IRI model. The change in density with latitudes for a fixed height is negligibly small as compared to that along a field line as seen from IRI model. The extent of the field line has been restricted to $200 \mathrm{~km}$. $\left(h_{\text {min }}\right)$ of ionospheric height, where the boundary conditions had been enforced on the eigen functions. At this point, it is important to discuss the role played by the boundary conditions. For analytic simplicity, it is customary to use the infinitely conducting ionosphere as the boundary that leads to negligible field line displacement and hence, the negligible electric field at the foot of the field line, i.e. field lines are rigidly tied at their ends in the ionosphere. Newton et al. (1978) looked at the effect of finite conductivity at the ionospheric boundary. They found that the characteristic period of oscillation (which is our primary concern here) is not significantly altered, but there can be measurable discipation of wave energy at the foot of the field line. The other extreme is to use the infinitely resistive ionospheric condition 


\section{FUNDAMENTAL TOROIDAL MODE FREQUENCY}

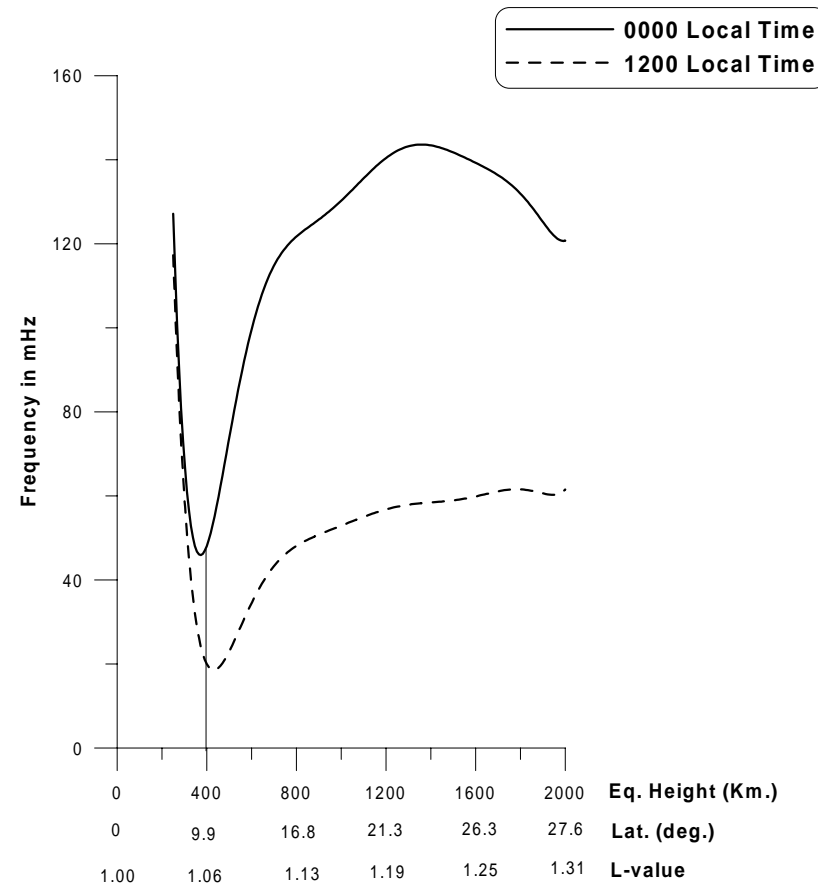

Fig. 1. Fundamental toroidal frequency as a function of equatorial height. Corresponding geomagnetic latitudes and L-values are marked. The period of oscillation for the field line with an equatorial height of $400 \mathrm{~km}$ has been shown by the vertical line.

that will lead to a large displacement in the foot of the field line and the electric field, and new standing modes are possible with a free-ended boundary condition at the end of the field line (Newton et al., 1978). In this case, the first mode that can produce significant oscillations of the field lines in the equatorial region is the second harmonic.

The time periods and hence, the frequencies of the fundamental toroidal oscillation of different field lines with a height ranging from $200 \mathrm{~km}$ to $2000 \mathrm{~km}$ (and hence, the different corresponding latitudes and L-value) have been calculated. The results thus obtained have been compared to that of Poulter et al. (1988).

It was seen that the typical frequency in the low-latitude region is of the order of $50 \mathrm{mHz}$ during nighttime and $20 \mathrm{mHz}$ during daytime. In the present analysis, we have used the data taken from the IRI model, whereas Poulter et al. (1988) estimated the fundamental toroidal eigen mode frequency in the global perspective self-consistently. The estimated eigen frequency is in excellent agreement with that of Poulter et al. (1988) and that validates our estimates. This calculation was necessary in order to obtain an order of magnitude estimate of the frequency to have a fair idea of the periodicity to be identified in the data. The estimated fundamental toroidal frequency, as a function of equatorial height, has been shown in Fig. 1 for 23 March 1999 at noon and midnight conditions. We note that the field lines in the ionospheric region have a characteristic period of 20-30 s. The characeristic period of

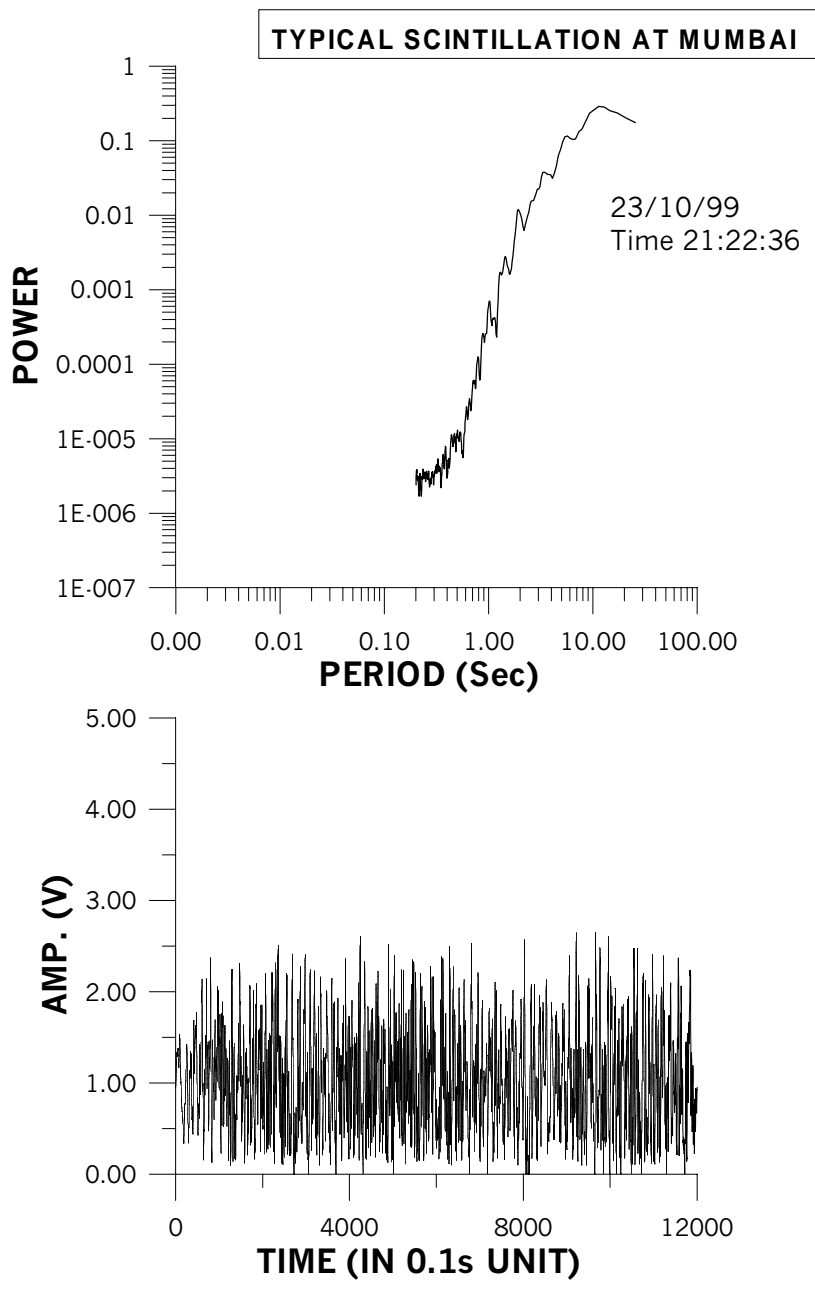

Fig. 2. The lower panel shows a typical scintillation at Mumbai, and the upper panel displays the corresponding power spectrum showing that field line eigen-period ( $\sim 25 \mathrm{sec}$ ) falls well beyond the Fresnel zone.

oscillation of the field line at a $400 \mathrm{~km}$ altitude at the equator is marked in Fig. 1 by a vertical line. We expect the field lines to preferentially oscillate at these periodicities if they are endogenously excited by the background turbulance.

Our next aim is to look for the signatures of such periodicities in the ionospheric scintillations recorded on a $250 \mathrm{MHz}$ radio beacon at the low-latitude stations. This will bee discussed in Sect. 3.

\section{Data analysis}

We examine the $250 \mathrm{MHz}$ radio wave signals received from the geostationary satellite FLEETSAT (at $73^{\circ} \mathrm{E}$ ). These are continuously monitored at Pondicherry, Mumbai and Ujjain. Digital data acquisition systems with a $10 \mathrm{~Hz}$ sampling rate have been used for a certain time period during 1999. The typical scintillation of the radio wave and the corresponding power spectrum have been shown in Fig. 2. It is evident from Fig. 1 that typical eigen mode frequency in the low- 


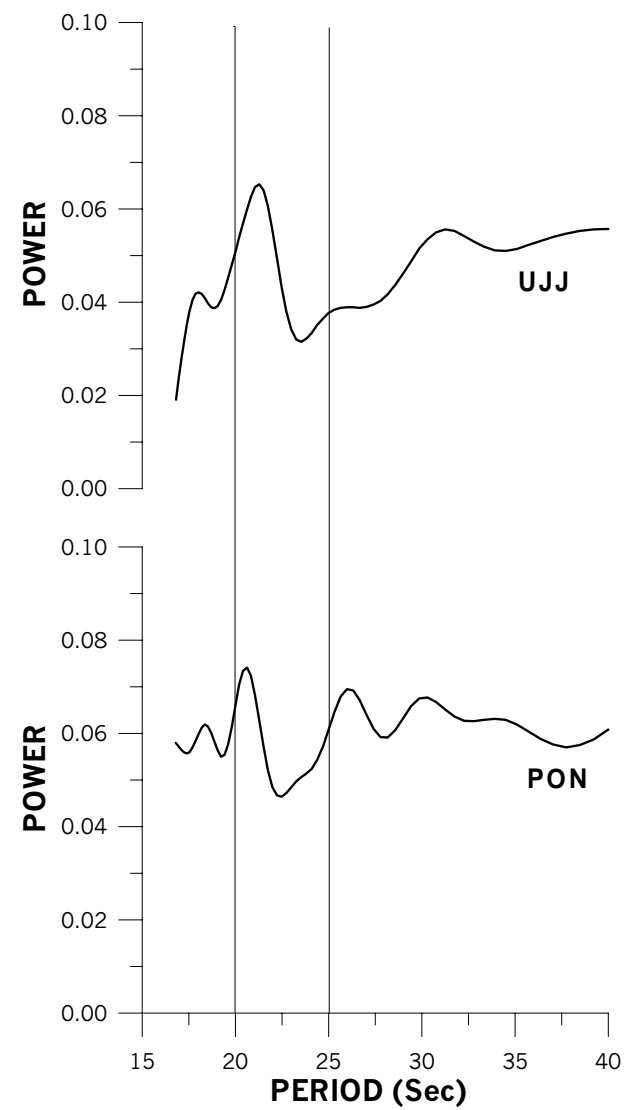

Fig. 3. Power spectrum of the $2 \mathrm{~s}$ averaged filtered scintillation on the specified day, at local time and at the specified stations. The plot shows the spectral peaks clearly standing out around the eigenperiod. The range of the eigen-period has been marked by the interval between two vertical lines.

latitude region of the Indian sector at nighttime is of the order of $50 \mathrm{MHz}$, which lies well outside the Fresnel range characterized by the spatial scale size $\sqrt{2 \lambda Z}$ and the time-scale $\sqrt{2 \lambda Z} / V_{o}$, where $\lambda$ is the wavelength of the radio wave, $Z$ is the height of the irregularity layer and $V_{o}$ is the transverse drift speed of the irregularities. Taking $Z$ at $\sim 400 \mathrm{~km}$ and $V_{o}$ at $\sim 150 \mathrm{~m} / \mathrm{s}$ (Pathan and Rao, 1996), spatial and time scales characterizing the Fresnel zone for $250 \mathrm{MHz}$. The radio waves are of the order of $1 \mathrm{~km}$ and $1 \mathrm{~s}$, respectively. These scales characterize the irregularities responsible for the coventional scintillation of the radio wave caused by small-scale irregularities (Bhattacharyya and Rastogi, 1991).

In the present analysis, our aim is to look for the characteristic period of field line oscillations in the scintillations of radio waves recorded at the low-latitude stations of Pondicherry, Mumbai and Ujjain. The events chosen for the analysis were those on the nights of 19 March 1999 and 7 October 1999. These were geomagnetically quiet days. We analyzed Pondicherry and Ujjain for 7 October and Pondicherry, Mumbai and Ujjain for 19 March. Data at the three stations Pondicherry, Mumbai and Ujjain recorded at $0.1 \mathrm{~s}$ were first averaged for $2 \mathrm{~s}$ to remove the high frequency scin-

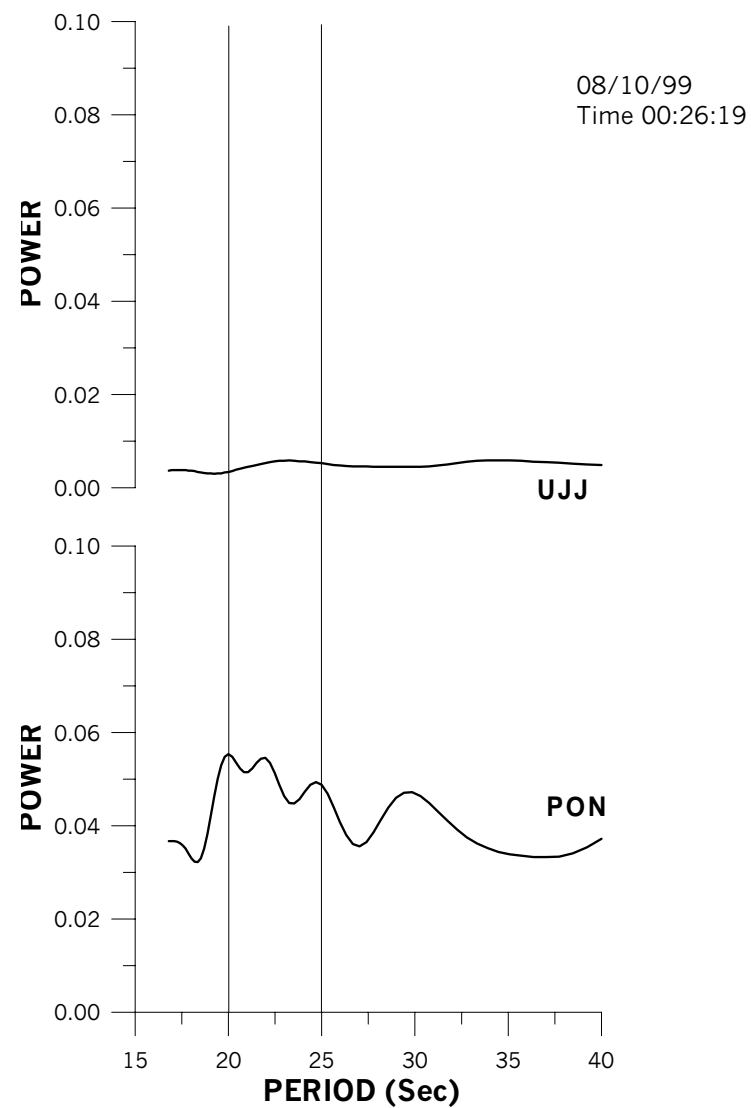

Fig. 4. Power spectrum of the $2 \mathrm{~s}$ averaged filtered scintillation on the specified day, at local time and at the specified stations. The plot shows a broader spectral peak around the eigen-period at Pondicherry, but does not show any such peak at Ujjain. The range of the eigen-period has been marked by the interval between two vertical lines.

tillations. The time series thus obtained were filtered using a zero-phase shift sixth order Butterworth type "band pass" filter for the period range of 10-100 s (Otnes and Enochson, 1978). The amplitude spectra were computed by direct Fourier transform, with amplitudes smoothed by the sliding window method over 5 points. The result thus obtained has been discussed in the following paragraph.

The results of the analysis have been presented in Figs. 3 to 8 . Figures 3 and 4 are the power spectra of simultaneous data collected at the two low-latitude stations of Pondicherry and Ujjain at local time 22:23:21 and 00:26:19, respectively, on the night of 7 October 1999, and Fig. 5 shows the scintillation record of the radio waves at two stations for the local time segment corresponding to Fig. 4. Similarly, Figs. 6 and 7 are the power spectra of simultaneous data collected at the three stations of Pondicherry, Mumbai and Ujjain at local time 21:36:14 and 22:36:42, respectively, on the night of 19 March 1999, and Fig. 8 shows the scintillation record of radio waves at the three stations at the local time segment corresponding to Fig. 7. From Figs. 3, 4, 6 and 7, it can be seen that spectral peaks near the characteristic eigen-period 


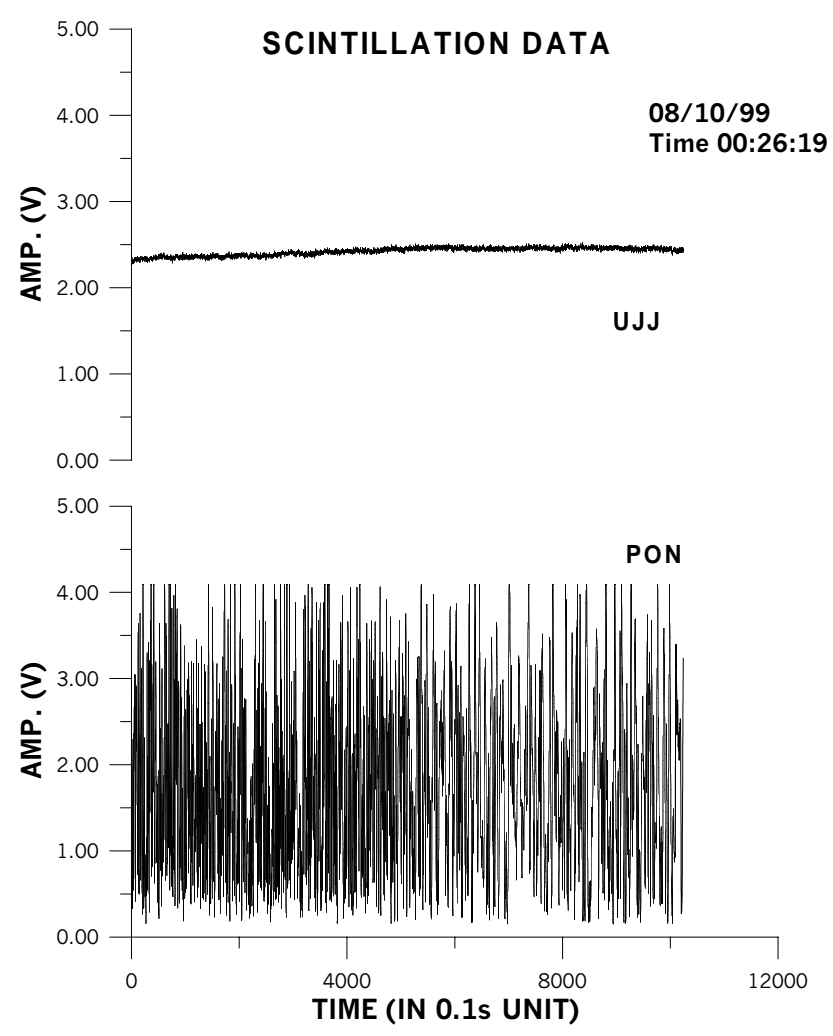

Fig. 5. Scintillations at the same time as specified in Fig. 4. The plot shows quite a significant scintillation at Pondicherry, whereas for Ujjain, the scintillations are not so significant.

clearly stand out for the analyzed events. A slight shift in frequency could be due to a small latitudinal difference and various statistical reasons which may not be significant for the purpose of the present study. Peaks are also seen at higher periods and do not consistently match at the stations, used for a particular event. The reasons for these peaks are not obvious and may be associated with different ambient velocities and their combinations present in the ionosphere. The elaboration of this point will be made in the next section.

In Fig. 4, the peak at the characteristic eigen-period is seen for Pondicherry, whereas no such peak is present for Ujjain, where the scintillation is not significant, as depicted by Fig. 5. Similarly, in Fig. 7 the peaks are seen at the characteristic eigen-period for Pondicherry and Mumbai, whereas no such peak is seen for Ujjain, where the scintillation is not significant, as demonstrated in Fig. 8. Ujjain, which is at a higher latitude as compared to Pondicherry and Mumbai, might not see the scintillations of radio waves if the irregularities are present at lower heights, since the radio waves will not be encountering the irregularities over Ujjain. Therefore, it is quite possible and physically plausible that the station situated at a higher latitude might not see the scintillations, whereas those situated at lower latitudes might see the same.

To confirm the absence of a peak at the characteristic eigen-period when the scintillations were not significant, we picked up many quiet time events with no significant scin-

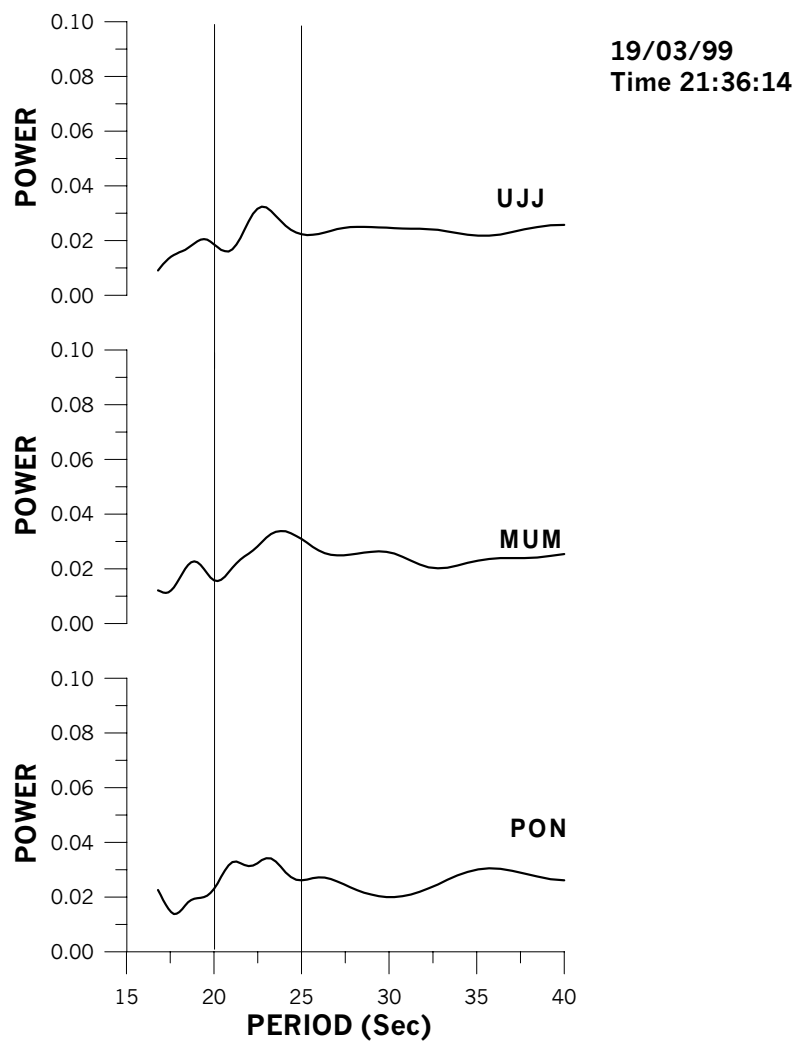

Fig. 6. Power spectrum of the $2 \mathrm{~s}$ averaged filtered scintillation on the specified day at local time and at the specified stations. The plot shows the spectral peaks clearly standing out around the eigenperiod. The range of the eigen-period has been marked by the interval between two vertical lines.

tillations and the data were subjected to the identical analysis. None of the spectra showed any significant peak at the characteristic eigen-period. This is consistent with the possibility that broad band energy available in the ionospheric scintillations might be responsible for driving the field line oscillations locally.

The field line oscillations should also manifest themselves through magnetic field variations at least at ionospheric heights. If some of the magnetic field variations reach the ground, it would provide added justification in favour of the field line oscillation hypothesis. Since the oscillations are around $20-25 \mathrm{~s}$ periodicity, a sampling rate of 1 or $2 \mathrm{~s}$ is desirable. Unfortunately, only data from standard magnetograms were available during most of the events and the provide no clue about the oscillations in the period range of our interest. Data at a $5 \mathrm{~s}$ sampling were available at Podicherry only very recently.

To support the above observational results, we have analyzed the $\mathrm{H}$ and $\mathrm{D}$ variations recorded at Pondicherry at a sampling rate of $5 \mathrm{~s}$ along with the simultaneous scintillation data of Tirunalvely for the night of 29 June 2000. It was a night of quiet magnetic variation and strong scintillation. No radio wave data were available from Pondicherry on that day. Radio wave signals with a sampling interval of $0.1 \mathrm{~s}$ had 


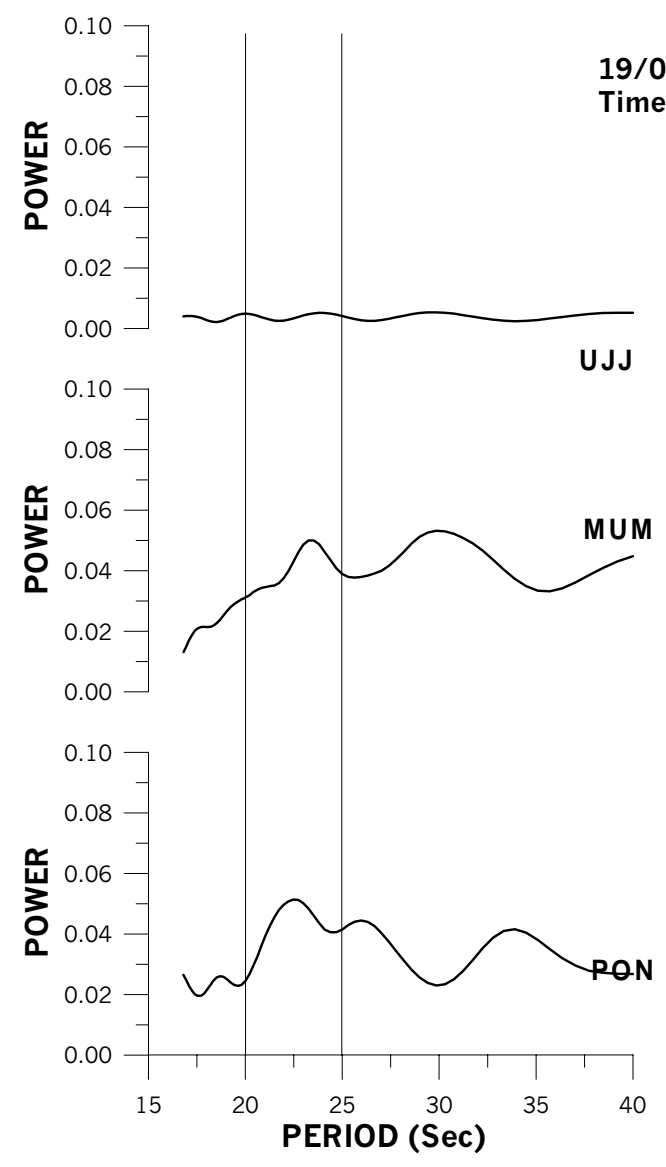

Fig. 7. Power spectrum of the $2 \mathrm{~s}$ averaged filtered scintillation on the specified day, at local time and at the specified stations. The plot shows spectral peaks around the eigen-period at Pondicherry and Mumbai, but does not show any such peak at Ujjain. The range of the eigen-period has been marked by the interval between two vertical lines.

to be averaged over $5 \mathrm{~s}$. The averaged radio wave data and the time series of the two vector components of the magnetic data, each consisting of 1024 points, were subjected to identical power spectrum analysis after band passing each series through a $10-100 \mathrm{~s}$ filter. The results are presented in Figs. 9 and 10. These results show significant peaks at the characteristic eigen-period. The spectral peaks occur at about the same period in the radio scintillations data and in the ground magnetic data.

The occurance of the peaks in the modulation of the radio wave signal and in the D-variations in the same period range is consistent with the field line oscillation hypothesis. The appearance of a similar variation in $\mathrm{H}$ could be attributed to the fact that compressive modes are automatically generated as the forcing is not zonally symmetric (Southwood and Hughes, 1983). Furthermore, the conductivity along the geomagnetic field line is very large and the D-variations are expected to be fully screened from the ground close to the geomagnetic equator (Roy, 2000). It is expected that at an ionospheric height, D-variations could have been much

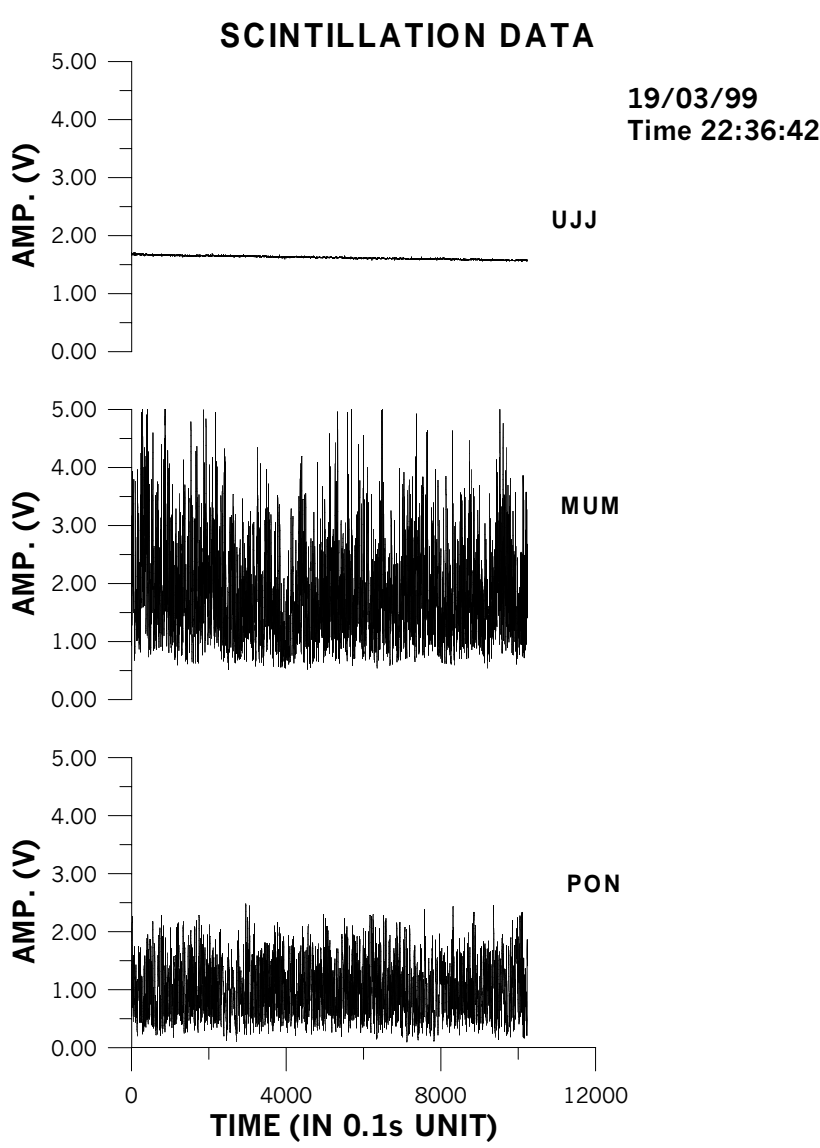

Fig. 8. Scintillations at the same time as specified in Fig. 7. The plot shows quite a significant scintillation at Pondicherry and Mumbai, whereas for Ujjain, the scintillations are not so significant.

larger than $\mathrm{H}$-variations and the oscillations could have been almost transverse.

We would like to emphasize that the phenomenon we are looking at probably originates at low-latitudes (within $\pm 20^{\circ}$ of the equator) and may not be directly associated with the Equatorial Electrojet (EEJ), which is confined to a much narrower latitudinal region. We are looking at a nighttime phenomenon that will not be affected by the EEJ.

\section{Numerical experiment}

The radio wave scintillations generated by ionospheric irregularities is conventionally examined within the gambit of the phase screen approximation (Booker and Majidiahi 1981, Bhattacharyya 1999). Here the role of the ionosphere is to introduce a phase shift on the transionospheric radio waves. The phase screen is characterized by the spatial characteristic which is, in turn, defined by the spatial structures of the irregularities in the ionosphere.

There are two basic processes considered for reproducing the observed scintillations on the ground. The first of these regards small-scale irregularities as the main source of the 


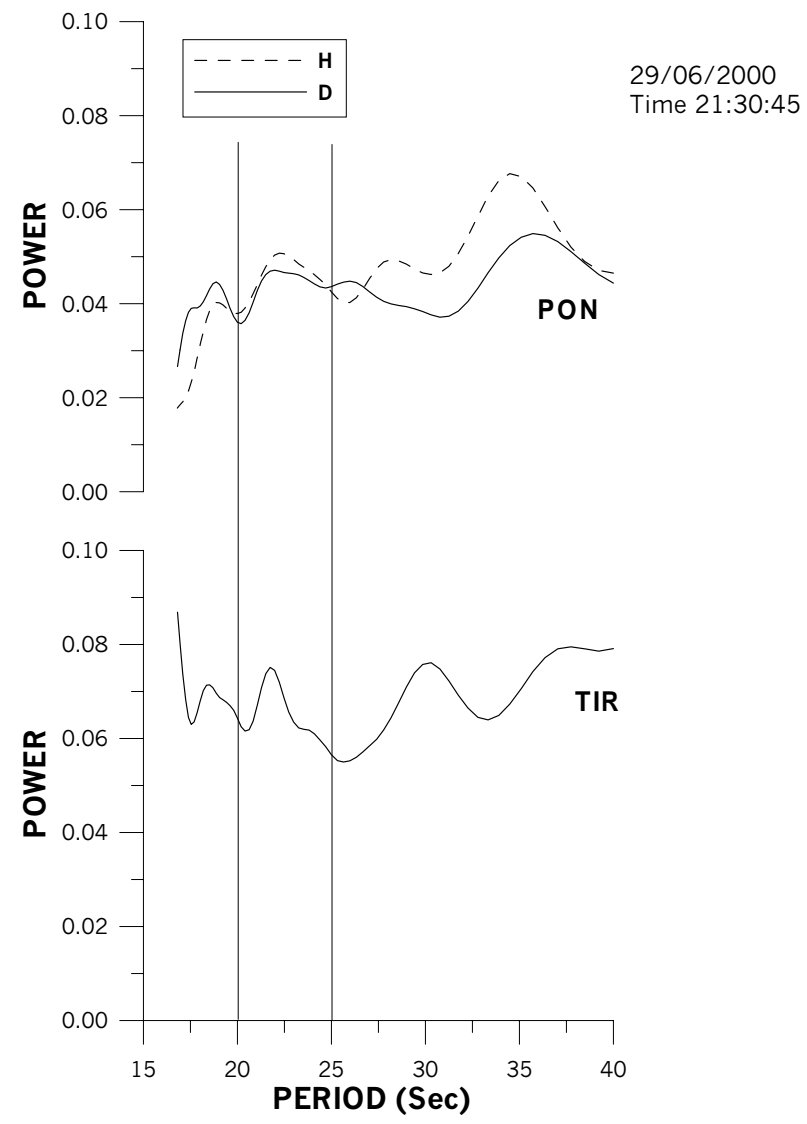

Fig. 9. The lower panel shows the power spectrum of $5 \mathrm{~s}$ averaged filter scintillations at Tirunalvely on the specified day and at local time. The dashed and solid curves in the upper panel respectively, show the power spectrum of $\mathrm{H}$ - and D-variations of ground magnetic data of $5 \mathrm{~s}$ sampling interval at Pondicherry. The range of the eigen-period has been marked by the interval between two vertical lines.

scintillations. The characteristic scale, in this case, is defined by $F=(\lambda z / 2 \pi)^{0.5}$ (where $\lambda$ is the wavelength and $z$ is the distance of the phase screen from the ground plane) and scale sizes less than the characteristic scale are believed to contribute to the observed scintillations despite the fact that the phase shifts introduced are of the order of 1 radian or less. The actual diffraction pattern generated on the ground is due to the superposition of waves coming out of a small area on the phase screen. The movement of the pattern on the ground due to the corresponding motion of the ionospheric medium generates the temporal scintillations observed at a fixed station.

A second view looks at large scale irregularities with a scale size typically of the order of the ionosphere's scale size which introduce a phase shift of 100-1000 radians. These can act as a refracting media, causing the spatial pattern on the ground due to the interference of refracted waves. The movement of these patterns might be reflected in terms of scintillation at a fixed location on the ground (Booker and Majidiahi, 1981). Modulation of radio wave signal strength

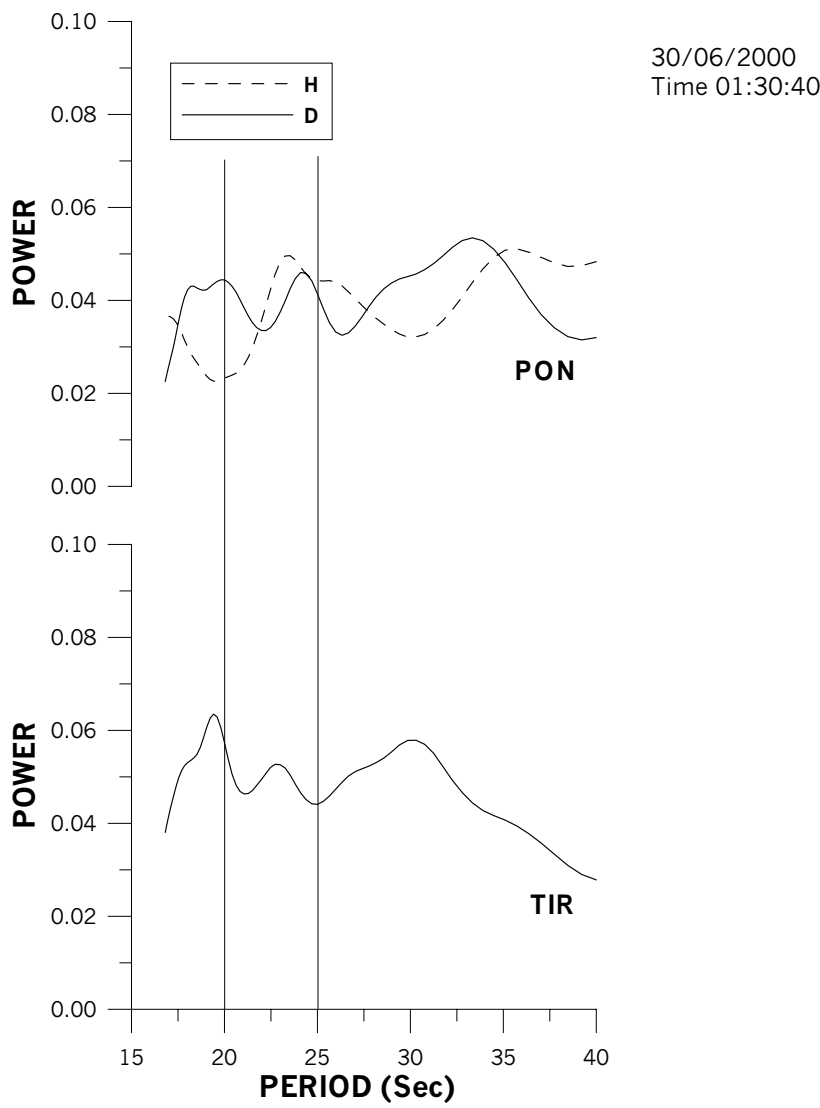

Fig. 10. The lower panel shows the power spectrum of $5 \mathrm{~s}$ averaged filter scintillations at Tirunalvely on the specified day and at local time. The dashed and solid curves in the upper panel, respectively, show the power spectrum of $\mathrm{H}$ - and D-variations of ground magnetic data of $5 \mathrm{~s}$ sampling interval at Pondicherry. The range of the eigen-period has been marked by the interval between two vertical lines.

due to the variation of the refractive index caused by imposed ULF waves is important at frequencies around $10 \mathrm{MHz}$ or less (Sutcliffe and Poole, 1990) but not at $250 \mathrm{MHz}$. It is the drift of the scattering that will have the most significant role to play. This follows from the fact that the refractive index at such a high sounding frequency is close to unity and almost independent of the background electron density or magnetic field.

For the suitable combination of the phase amplitude and the scale size of the phase variation, the refracted radio waves may focus close to the plane of the ground (Titheridge, 1971). Our results seem to indicate that the field line eigenperiod is much larger than the main body of scintillations conventionally associated with spatial scales less than the Fresnel scale. Furthermore, the shorter scale sizes have shorter lifetime and may not be coherent over the period of a number of field line oscillations. An appreciable variation in the radio wave signal can be produced as a result of even a small movement in the irregularity pattern in the ionosphere. Our interest here is to see via a simple numerical experiment 
if such movement provided by transverse field line oscillations can explain such a large-scale variation in light of refractive scattering. If so, such variations can be identified as the signatures of field line oscillations imprinted on the radio wave scintillations. This will provide additional evidence in support of field line oscillations.

We consider an irregularity which imposes a sinusoidal phase shift, $\phi$, varying in the $x$-direction (westward), on a downward moving wave of unit amplitude. The phase shift is given as

$\phi=\phi_{0} \cos \frac{x-x_{0}}{d}$,

where $\phi_{0}$ is the amplitude of the phase variation, $x_{o}$ is a reference point corresponding to the maximum phase shift, $x$ is the location of the point from where the radio wave leaves the ionosphere and $d$ is the scale size of the irregularity. It is assumed that the irregularities are infinitely long in the $y$ direction. We assume that the pattern moves as a whole with a mean drift velocity, $V_{o}$, and an additional motion, $\Delta V$, associated with a field line oscillation. $x$ is fixed, and $x_{o}$ changes with time, so that $x-x_{o}$ becomes an explicit function of time. This motion is the reason that the incoming wave reaching the observer moves through various sections of the irregularity and in the process, the radio wave that reaches a fixed point on the ground undergoes a time dependent phase change, given by Eq. (6). The effects of the time dependent phase variations are observed as scintillations on the ground. Considering $V_{o}$ as the mean drift velocity of the pattern and $\Delta V$ as an additional motion associated with a field line oscillation, after time $t, x-x_{o}$ can be written as

$x-x_{0}=V_{0} t+\frac{\Delta V}{\Omega_{r}} \sin \Omega_{r} t+K$,

where, $\Omega_{r}$ is the characteristic angular frequency and $\Delta V$ is the velocity of the oscillation of the field line and $K$ denotes the distance of the radio wave from the point corresponding to maximum phase shift at $t=0$. From our model calculation, we note that the amplitude of the field line motion decreases from $8.5 \mathrm{~m} / \mathrm{s}$ to $1.4 \mathrm{~m} / \mathrm{s}$ as we move $10^{\circ}$ away from the equator along a field line cutting the equator at $500 \mathrm{~km}$. This is for the toroidal mode of oscillation corresponding to a $1 \mathrm{nT}$ fluctuation in the magnetic field. This change in velocity due to the oscillation of the field lines is quite significant and implies that the amplitude of the phase oscillations introduced varies with latitude.

A radio wave normally incident on the phase varying screen, representing the vertically integrated effects of phase shifts introduced by the spatially inhomogeneous ionospheric irregularities, is refracted by a small angle, $\epsilon$, given by (Titheridge, 1971)

$\epsilon=\frac{\lambda}{2 \pi} \frac{d \phi}{d x}$.

The wave leaving the ionosphere at the horizontal coordinate $x$ touches the ground at a horizontal coordinate $y$ given by

$y=x+h \epsilon$,

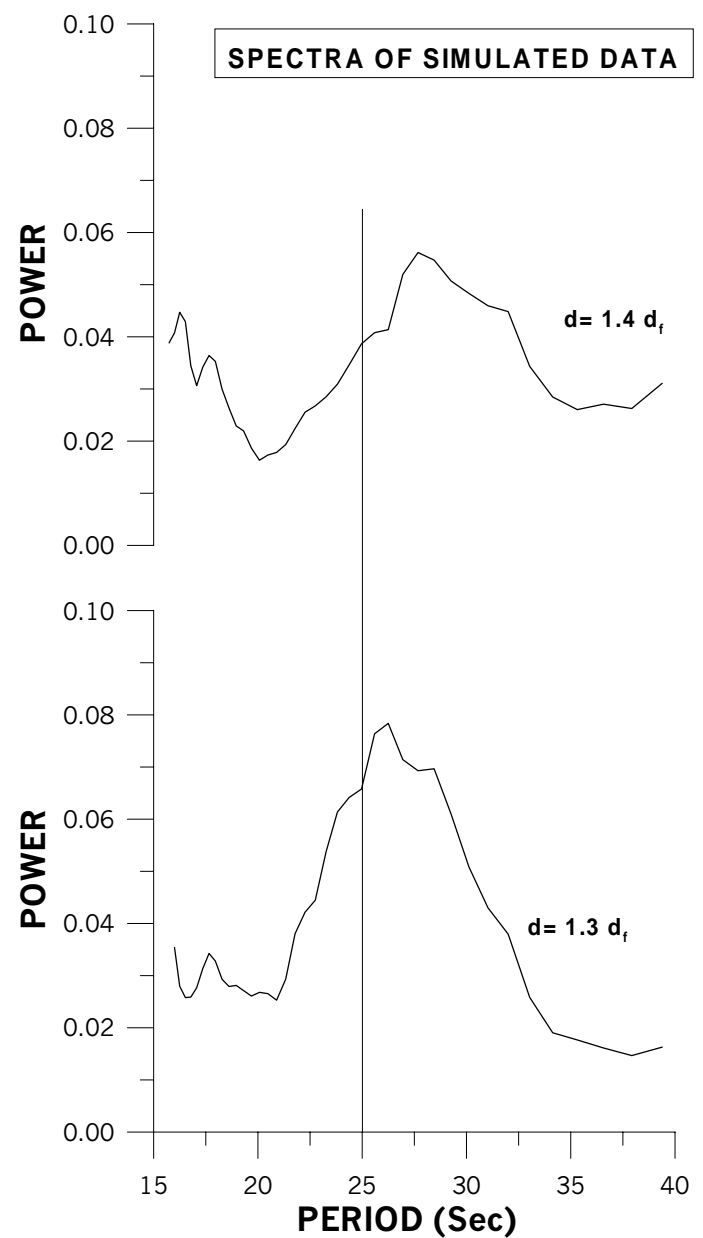

Fig. 11. Power spectrum of the numerically simulated scintillation data at a $2 \mathrm{~s}$ sampling interval. The plot has been shown for $d>d_{f}$. The lower panel shows the spectrum for $d=1.3 d_{f}$ and the upper panel shows the same for $d=1.4 d_{f}$. The characteristic eigenperiod, used for the nomerical calculation, has been marked by the vertical line.

where $h$ denotes the height of the irregularity layer.

The amplitude $A$ and phase $\Psi$ of the wave as it reaches the point $y$ on the ground is given as (Titheridge, 1971)

$A=\left(\frac{d y}{d x}\right)^{-1 / 2}=\left(1+h \frac{d \epsilon}{d x}\right)^{-1 / 2}$,

$\Psi=\phi-\pi h \epsilon^{2} / \lambda$.

It is obvious from Eq. (10) that as $d y / d x$ approaches zero, the amplitude tends to infinity. It is not difficult to understand why this happens. $d y /(d x) \rightarrow 0$ implies that the waves emanating from the entire neighbourhood of the point $x$ in the ionosphere reach or more correctly converge to the point $y$. This leads to the focussing of the beam. The singularity, of course, will not occur in a real physical situation because the diffraction of the ray smears out the signal around $y$ and this results in a finite (but large) wave amplitude at $y$ (Uscinski, 1971). It should be remembered that we are looking at 
the phenomenon of refraction using the ray theory, ignoring small-scale effects associated with diffraction. The focussing of the beam occurs when

$\frac{d y}{d x}=1-\frac{h \lambda}{2 \pi} \frac{\phi_{0}}{d^{2}} \cos \left(\frac{x-x_{0}}{d}\right)=0$.

The necessary condition for this to happen is

$\frac{h \lambda}{2 \pi} \frac{\phi_{0}}{d^{2}} \geq 0$.

For a fixed $\phi_{0}$, focussing will occur if the scale size of the irregularity is less than $d_{f}$ given by

$d_{f}=\sqrt{\frac{h \lambda \phi_{0}}{2 \pi}}$.

Given $\phi_{0}$, if the scale length is less than $d_{f}$, a more complex structure will occur. We concentrate here on a scale size close to, but greater than $d_{f}$ for the sake of simplicity.

We are interested in tracking the time variation at a fixed ground station (i.e. for fixed $y$ ). We note from Eqs. (6) and (7) that as the irregularity pattern moves, $x$ and $\phi$ becomes an explicit function of time and so does $\epsilon$ given by Eq. (8). The wave received at $y$ emanates from a different point (or points) $x$ in the ionosphere as the irregularity pattern drifts. The first step is to determine $x(t)$ at each instant of time from Eqs. (8) and (9) using an iterative algorithm. Once $x$ is determined, the amplitude and phase can be evaluated from Eqs. (10) and (11). The signal $W(t)$ received on the ground is given by

$W(t)=A \cos \Psi$.

After arriving at a $\phi_{0}$ and $d$, we generate a time series of $W(t)$ of 1024 points at $2 \mathrm{~s}$ sampling interval and perform a spectral analysis of the resultant time series after passing it through a band pass filter that only allows for 10 to $100 \mathrm{~s}$ periodicities. The amplitude spectrum was computed by direct Fourier transform and the amplitudes were smoothed by a sliding window method over 5 points. It should be noted that identical analysis was performed while dealing with observed scintillation data.

The experiment was performed for various combinations of $\phi_{o}$ and $d$ for different h (300-500 km), $V_{0}(100-200 \mathrm{~m} / \mathrm{s})$ and $\Delta V(10-20 \mathrm{~m} / \mathrm{s})$, taking $\lambda=1.2 \mathrm{~m}$ (corresponding to a $250 \mathrm{MHz}$ radio wave) and $T_{r}=25 \mathrm{~s}$ (characteristic eigenperiod). The results presented here is for $h=300 \mathrm{~km}$. For our presentation, at this height, we have used $V_{0}=150 \mathrm{~m} / \mathrm{s}$ based on Pathan and Rao (1996). $\Delta V=20 \mathrm{~m} / \mathrm{s}$ is taken by choice and it does not make any qualitative difference in the result. The choice of $\phi_{o}$ determines $d_{f}$. We cannot take $d=d_{f}$ as the scale size since that will lead to singularity and hence, we have chosen $d$ close to $d_{f}$. The scale size of the irregularities could be from submeters to hundreds of kilometers (Hysell, 2000). As we are interested in large-scale irregularities that can only be obtained by a large amplitude of the phase variation $\phi_{o}$, we chose $\phi_{o}=2000$ radians such that $d_{f} \sim 10 \mathrm{~km}$.

The spectrum thus obtained shows a clear-cut peak standing out around the eigen-period, as demonstrated in Fig. 11.
The results obtained from this preliminary numerical experiment appear to reproduce the essential features of the power spectra near the characteristic eigen-period obtained from the observational data. In Fig. 11, the results are shown for the cases where the scale size of the phase variation has been taken to be slightly greater than that required for focussing $\left(d>d_{f}\right)$. It is seen from the spectra that the peaks do not fall exactly on the characteristic period (marked by the vertical line in Fig. 11), but become slightly shifted. The shift is more for $d\left(d=1.4 d_{f}\right)$ farther from $d_{f}$, compared to the nearer one $\left(d=1.3 d_{f}\right)$. In addition, it should be noted that the power of the peak around the characteristic period is less for $d=1.4 d_{f}$, compared to that for $d=1.3 d_{f}$.

To obtain an insight into what is happening, the following simplified analytic treatment can be useful. For $\epsilon<1$, we retain only linear terms in the expansion of the expression in Eqs. (8) to (12).

Assuming that $\Delta V /\left(\Omega_{r} d\right)<1$, Eqs. (10) and (11) take the form

$$
A=1+\frac{h \lambda \phi_{0}}{4 \pi d^{2}}\left[\cos \frac{V_{0} t}{d}-\frac{\Delta V}{\Omega_{r} d} \sin \left(\Omega_{r} t\right) \sin \frac{V_{0} t}{d}\right]
$$

and

$\Psi=\phi_{o}\left[\cos \frac{V_{0} t}{d}-\frac{\Delta V}{\Omega_{r} d} \sin \left(\Omega_{r} t\right) \sin \frac{V_{0} t}{d}\right]$.

For the chosen $V_{0}$ and $d$, the first term in the square bracket in Eqs. (14) and (15) oscillates with a frequency of the order of $1 \mathrm{mHz}$, which is much smaller than the frequency of the oscillation $(\sim 40 \mathrm{MHz})$ of the second term and hence, will act almost as a DC. It is the second term that gives the combinations of frequencies $\Omega_{r}$ and $V_{0} / d$ in both the amplitude and the phase. It should be noted that they can combine in both constructive and destructive ways, resulting in peaks at lower $\left(V_{0} / d-\Omega_{r}\right)$ and higher $\left(V_{0} / d+\Omega_{r}\right)$ frequencies as compared to the characteristic frequency, $\Omega_{r}$. In a real data situation, there could be various time scales that could combine with the ambient motion to give rise to various periodicities observed in the actual spectrum.

This small numerical exercise shows that the refractive scattering could be a plausible mechanism. It can provide the explanation for the observed peak at the characteristic period due to the movement in the large-scale ionospheric irregularities provided by the local field line oscillations.

\section{Results and conclusions}

Theoretical estimates of eigen-frequencies at various latitudes shows that in low-latitude regions, the magnitudes of the frequency are typically around $50 \mathrm{mHz}$ and $25 \mathrm{mHz}$ during midnight and noon, respectively. This has been shown in Fig. 1. The calculation is based on the various assumptions stated in detail in Sect. 4. This calculation was done in order to have an order of the estimate of the eigen-frequency at low-latitudes. A similar type of model calculation was 
done by Poulter et al. (1988) in a self-consistent way with a global perspective in mind by taking a specific (but nonstandard) ion density model. Our model uses the standard IRI ion density model and the results obtained are in excellent agreement with those obtained by Poulter et al. (1988). This shows that the eigen-periods are quite stable and they do not depend much on the model.

The peak at the eigen-period is consistently seen in the spectra of the analyzed scintillation data, as depicted by Figs. 3, 4, 6 and 7. The implication of such peaks is understood in terms of the modulation of the downward moving radio waves by field line oscillations. The very purpose of the present investigation was to look for such a modulation at low-latitude stations. The reasons for the peaks at higher periods are not obvious and may be due to a combination of velocities associated with ionospheric irregularities.

No such peak is seen for the events when there is no scintilltion or the scintillations are very weak. Such events are shown in Figs. 5 and 8 and the corresponding spectra in Figs. 4 and 7, respectively. This result suggests that broad band energy available in ionospheric irregularities might be playing some role in exciting field line oscillations at lowlatitudes. This is why we do not see the effect of field line oscillations in the spectra when there is no scintillation or the scintillation is very weak.

In Sect. 4, we have made an attempt to explain this phenomena by a simple numerical experiment. We generated a time series in light of refractive scattering by taking into account the effect of field line oscillations. The spectra of the time series have been shown in Fig. 11 and they are consistent with those obtained from the observational data. The presence of peaks at the eigen-period in the spectra of a theoretically generated time series leads us to believe in the process of the modulation of downward moving radio waves by field line oscillations. This is only a possible explanation and more work needs to be done to gain a firmer footing. Our observations do support the idea of Saito (1983) that endogenous forces may be exciting field line oscillations in the lowand equatorial-latitudes. The results from ground magnetic data also seem to support the arguement.

From an energetics point of view, it may be noted that instabilities responsible for the Equatorial Spread-F (ESF) generate plasma density irregularities with a scale size ranging from centimeters to hundreds or perhaps thousands of kilometers and relative amplitudes in excess of 99\% (Hysell, 2000). The classical radio wave scintillations open a window to every narrow sector of these oscillations. The total energy available in the irregularities is contributed by larger scale oscillations. 'Bubbles' associated with the irregularities often represent an order of magnitude of fluctuations in the electron density. Bulk motion as high as the $\mathrm{km} / \mathrm{s}$ associated with the magnetic field fluctuations of over $100 \mathrm{nT}$ have been detected (Aggson et al., 1992). An estimate of the energy that can be discipated in the ESF region was made by Cole (1974). He showed that the energy released could be of the order of $10^{-10} \mathrm{~J} / \mathrm{m}^{3} / \mathrm{sec}$ or higher. The typical energy associated with a field line oscillation with a $1 \mathrm{nT}$ amplitude will be of the order of $10^{12} \mathrm{~J} / \mathrm{m}^{3}$. The energy available is thus adequate to excite field line oscillations with much larger amplitudes. The possible process of Alfvén wave generation has been described in literature (Aggson et al., 1992). We anticipate that the appropriate frequency of the oscillations should correspond to the natural period of field line oscillations. A mechanism of reflection of Alfvén waves at conjugate regions, as suggested by Bhattacharyya and Burke (2000) for the nonlinear evolution of the ESF, will also naturally lead to such oscillations.

The results obtained by us are preliminary in nature, but nevertheless encouraging as it involves an important tool for studying field line oscillations in low-latitude regions through the scintillations of the radio waves recorded on the ground. However, more detailed investigations are needed in order to understand the phenonomena in real physical terms. Once established, attempts could be made to study the coupling between the field line oscillations and the ionospheric irregularities in order to quantify the energy transfer needed to excite the oscillation and to know precisely about the physical conditions required for such oscillations to be excited.

\section{References}

Aggson, T. L., Burke, W. J., Maynard, N. C., Hanson, W. B., Anderson, P. C., Slavin, J. A., Hoegy, W. R., and Saha, J. L.: Equatorial bubbles updrafting at supersonic speeds, J. Geophys. Res., 97, 8581-8590, 1992.

Anderson, B. J., Engebretson, M. J., Rounds, S. P., Zanetti, L. J., and Potemra, T. A.: A statistical study of Pc3-5 pulsations observed by the AMPTE/CCE magnetic fields experiment 1 . Occurrence distributions, J. Geophys. Res., 95, 10495-10 523, 1990.

Bhattacharyya, A.: Deterministic retrieval of ionospheric phase screen from amplitude scintillations, Radio Sci., 34, 229-240, 1999.

Bhattacharyya, A. and Rastogi, R. G.: Structure of ionospheric irregularities from amplitude and phase scintillation observations, Radio Sci., 26, 439-449, 1991.

Bhattacharyya, A. and Burke, W. J.: A transmission line analogy for the development of equatorial ionospheric bubble, J. Geophys. Res., 105, 24 941-24 950, 2000.

Booker, H. G. and Majidiahi, G.: Theory of refractive scattering in scintillation phenomena, J. Atmos. Ter. Phys., 43, 1199-1214, 1981.

Budden, K. G.: Radio waves in the ionosphere, page 79, Cambridge University Press, London, 1961.

Cole, K. D.: Energetics of and a source of energy for equatorial spread-F events, J. Atmos. Ter. Phys., 36, 1099-1102, 1974.

Cummings, W. D., O’Sullivan, R. J., and Coleman, P. J.: Standing Alfvén waves in the magnetosphere, J. Geophys. Res., 74, 778793, 1969.

Dungey, J. W.: Electrodynamics of the outer atmospheres, Rep. 69 Ions. Res. Lab. Pa. State Univ., University Park, 1954.

Fedorov, E., Pilipenko, V., Surkov, V., Rao, D. R. K., and Yumoto, $\mathrm{K}$.: Ionospheric propagation of magnetohydrodynamic disturbances from the equatorial electrojet, J. Geophys. Res., 104, 4329-4336, 1999. 
Hysell, D. L.: An overview and synthesis of plasma irregularities in equatorial spread-F, J. Atmos. Ter. Phys., 62, 1037-1056, 2000.

Menk, F. W., Waters, C. L., Ziesolleck, C. W. S., Fraser, B. J., Lee, S. H., and McNabb, P. W.: Ground measurements of low-latitude magnetospheric field line resonances, in Solar wind sources of magnetospheric ULF waves (Eds) Engebretson, M. J., Takahashi, K., and Scholer, M., Geophys. Mono., 81, AGU, Washington D. C., 299-310, 1994.

Newton, R. S., Southwood, D. J., and Hughes, W. J.: Damping of geomagnetic pulsations by the ionosphere, Planet. Sp. Sci., 26, 201-209, 1978.

Otnes, R. K. and Enochson, L.: Applied time series analysis, Vol. 1, Basic techniques, 449, Wiley-Interscience, New York, 1978.

Pathan, B. M. and Rao, D. R. K.: Seasonal and solar cycle association of zonal drifts of ionospheric plasma irregularitie in the Indian equatorial region, Ann. Geophyicae, 14, 297-303, 1996.

Poulter, E. M., Allan, W., and Bailey, G. J.: ULF pulsation eigenperiods within the plasmosphere, Planet. Space Sci., 36, 185-196, 1988.

Ratcliffe, J. A.: An introduction to the ionosphere and magnetosphere, Cambridge university press, London, 1972.

Roy, M.: Geomagnetic micropulsation and the equatorial ionosphere, J. Ind. Geophys. Union, 4, 1-6, 2000.

Saito, T.: Resonance model on Pc 3 in sobtropical region Contribuciones cientificas para conmemorar of 75 aniversario del Observatorio del Ebro, (Eds) Cardus, J. O. and Roquetes, S. I., 175$180,1983$.
Singer, H. J., Southwood, D. J., Walker, R. J., and Kivelson, M. G.: Alfvén wave resonances in a realistic magnetospheric magnetic field geometry, J. Geophys. Res., 86, 4589-4596, 1981.

Sinha, A. K. and Rajaram, R.: An analytic approach to toroidal eigen mode, J. Geophys. Res., 102, 17 649-17 657, 1997.

Southwood, D. J. and Hughes, W. J.: Theory of hydromagnetic waves in the magnetosphere, Space Sci. Rev., 35, 301-366, 1983.

Sutcliffe, P. R. and Poole, A. W. V.: Ionospheric Doppler and electron velocities in the presence of ULF waves, J. Geophys. Res., 94, 13 505-13 514, 1989.

Sutcliffe, P. R. and Poole, A. W. V.: The relationship between ULF geomagnetic pulsations and ionospheric Doppler oscillations: Model predictions, Planet. Space Sci., 38, 1581-1589, 1990.

Takahashi, K. and Anderson, B. J.: Distribution of ULF-energy $(\mathrm{f}<80 \mathrm{mHz})$ in inner magnetosphere: a statistical analysis of AMPTE CCE magnetic field data, J. Geophys. Res., 97, $10751-$ $10773,1992$.

Titheridge, J. E.: The diffraction of satellite signals by isolated ionospheric irregularities, J. Atmos. Sol. Terr. Phys., 33, 47-69, 1971.

Uscinski, B. J.: The element of wave propagations in random media, McGraw-Hill, London, 1977.

Yeh, K. C. and Liu, C. H.: Radio wave scintillation in the ionosphere, Proc. IEEE, 70, 324-359, 1982.

Yumoto, K.: Generation and propagation mechanism of lowlatitude magnetic pulsations - a review, J. Geophys., 60, 79-105, 1986. 